\title{
CO Gas and Dust Outbursts from Centaur 29P/Schwassmann-Wachmann
}

\author{
K. Wierzchos ${ }^{1}$ (1) and M. Womack ${ }^{2}$ (1) \\ ${ }^{1}$ Catalina Sky Survey, University of Arizona, Lunar and Planetary Lab, Tucson, AZ 85721, USA; kacperwierzchos@gmail.com \\ ${ }^{2}$ Florida Space Institute and Department of Physics, University of Central Florida, USA \\ Received 2019 September 25; revised 2020 January 16; accepted 2020 January 20; published 2020 February 28
}

\begin{abstract}
29P/Schwassmann-Wachmann is an unusual solar system object. Originally classified as a short-period comet, it is now known as a Centaur that recently transferred to its current orbit, and may become a Jupiter family comet. It has exhibited a dust coma for over $90 \mathrm{yr}$, and regularly undergoes significant dust outbursts. Carbon monoxide is routinely detected in high amounts and is typically assumed to play a large role in generating the quiescent dust coma and outbursts. To test this hypothesis, we completed two three-month-long observing campaigns of the $\mathrm{CO}$ $J=2-1$ rotational line using the Arizona Radio Observatory $10 \mathrm{~m}$ Submillimeter Telescope during 2016 and 2018-2019, and compared the results to visible magnitudes obtained at the same time. As the Centaur approached its 2019 perihelion, the quiescent dust coma grew 45\% in brightness, while it is unclear whether the quiescent CO production rate also increased. A doubling of the CO production rate on 2016 February 28.6 UT did not trigger an outburst nor a rise in dust production for at least 10 days. Similarly, two dust outbursts occurred in 2018 while $\mathrm{CO}$ production continued at quiescent rates. Two other dust outbursts may show gas involvement. The data indicate that $\mathrm{CO}$ and dust outbursts are not always well correlated. This may be explained if $\mathrm{CO}$ is not always substantially incorporated with the dust component in the nucleus, or if $\mathrm{CO}$ is primarily released through a porous material. Additionally, other minor volatiles or physical processes may help generate dust outbursts.
\end{abstract}

Unified Astronomy Thesaurus concepts: Centaurs (215); Comets (280); Small solar system bodies (1469); Short period comets (1452)

\section{Introduction}

Comets are icy bodies that contain well-preserved material from the early stages of the solar system formation. They formed at large heliocentric distances where temperatures were low enough for many ices to condense. Their nuclei contain trapped volatiles, refractory grains, and rock. When they get close enough to the Sun, ices sublimate and release gas and dust grains that form envelopes known as comae. Some of these grains have frozen ices on them, which may then sublimate once immersed in the solar radiation field. Measuring the composition and structure of cometary objects, and how they become active, provides important observational constraints to models of the early solar system (Mumma \& Charnley 2011; Mandt et al. 2015; Fulle et al. 2019).

Comets were historically classified into two broad categories based on their orbital periods: long-period $\left(P_{\text {orb }}>\right.$ $200 \mathrm{yr})$ and short-period comets. Long-period comets are distributed with random inclinations and large distances out to the Oort Cloud, whereas short-period comets tend to be found closer in and near the ecliptic, due to originating from the trans-Neptunian region of the Kuiper Belt. Since orbital interactions with the giant planets, especially Jupiter, play a large role in many comets' dynamical evolution, the Tisserand parameter $\left(T_{\mathrm{j}}=a_{\mathrm{J}} / a+2 \sqrt{a / a_{\mathrm{j}}\left(1-e^{2}\right)} \cos i\right)$ is also used to categorize comets (Levison \& Duncan 1994). Many cometary objects whose orbits are Jupiter-controlled undergo a transitional Centaur phase and eventually become Jupiter family comets (JFCs) and have $2<T_{\mathrm{J}}<3$ (Levison \& Duncan 1997; Dones et al. 2015; Sarid et al. 2019).

29P/Schwassmann-Wachmann (hereafter 29P/SW1) is a remarkable cometary object. It temporarily occupies a nearly circular orbit, slightly larger than Jupiter's, with an eccentricity of $e=0.043$, a semimajor axis of $a=6.02 \mathrm{au}$, and an inclination of $i=9^{\circ} .36$, according to the Minor Planet Center (epoch 2019 March 18.0).

Originally classified as a short-period comet $\left(P_{\text {orbital }}=14.6 \mathrm{yr}\right)$, dynamical studies show that $29 \mathrm{P} / \mathrm{SW} 1$ is a Centaur (with $T_{\mathrm{J}}=2.985$ ) that probably only recently transferred to its current location, a newly discovered "Gateway" transitional orbit to the JFC population, and may eventually become the brightest comet seen by humankind (Sarid et al. 2019). Thermal modeling of Spitzer-IRAC photometry predicts that the nucleus of $29 \mathrm{P} / \mathrm{SW} 1$ is much larger than a typical comet, with a radius of $32 \pm 2 \mathrm{~km}$ (Schambeau 2018), and is by far the largest of the four objects known to be occupying the Gateway orbit. 29P/SW1's nucleus is probably larger than all JFCs and in a 1999 survey, its absolute nuclear magnitude was found to be brighter than all other JFCs measured to date (Fernández et al. 1999).

29P/SW1 is one of $\sim 30$ known active Centaurs and is legendary for having a continuously present dust coma superimposed with explosive outbursts several times a year (see Table 1 and references on footnote (a)). The vast majority of its activity is documented with visible magnitudes, and most of the light at this wavelength comes from the reflection and scattering of sunlight off the dust particles in the coma, with smaller contributions from some ions and radicals (Cochran \& Cochran 1991). While small-scale changes in the lightcurve may be caused by nucleus rotation, large-scale changes in 29P/ SW1's visible magnitude are probably largely due to changes in quantity or types of dust production output, including explosive outbursts. Long-term tracking of 29P/SW1 with visible lightcurves show there is a slight preference for such outbursts to occur between perihelion and aphelion (Hughes 1975; Cabot et al. 1996; Krisandova \& Svoren 2014), with an average of about seven per year over the most recent orbit (Trigo-Rodríguez et al. 2008, 2010). It is intriguing to consider how much more dramatic its behavior may become a few 
Table 1

Typical Visual Magnitudes of 29P/SW1

\begin{tabular}{lcc}
\hline \hline Quantity & Quiescent & Outbursting \\
\hline$m_{v}{ }^{\mathrm{a}}{ }^{\mathrm{b}}$ & $\sim 17$ & $16-10$ \\
$m_{\text {helio }}{ }^{\mathrm{b}}$ & $\sim 13$ & $12-6$ \\
\hline
\end{tabular}

Notes.

${ }^{\text {a }}$ Quiescent and outbursting values of the visible magnitude reported for 29P, such as Roemer (1958, 1962), Whipple (1980), Jewitt (1990), Cochran et al. (1991), Trigo-Rodríguez et al. (2008), Ferrín (2010), Miles et al. (2016), Schambeau (2018), and this paper.

${ }^{\mathrm{b}} m_{\text {helio }}$ is the apparent magnitude, $m_{v}$, corrected for geocentric distance with Equation (1), and is also referred to as $m(1, r, \theta)$.

thousand years from now if its orbit takes it significantly closer to the Sun to become a JFC (Sarid et al. 2019).

We consider a dust outburst to be a brightening event of at least 1 mag of the nuclear magnitude (typically the central $5^{\prime \prime}-10^{\prime \prime}$ part of the coma) that takes place within a few hours to a day. Brightness variations occur on smaller scales, but this threshold definition for an outburst is consistent with the analysis of many other published data sets (Richter 1941; Roemer 1958; Trigo-Rodríguez et al. 2008, 2010; Miles et al. 2016; Schambeau et al. 2019). The dust coma can also present morphological changes as an outburst progresses (Schambeau et al. 2017) and outbursts are documented at longer wavelengths (e.g., Hosek et al. (2013). Not a great deal is known about the outburst characteristics, and there is very little observational evidence for possible triggers.

The ever-present dust coma is also remarkable, because at $\sim 6$ au from the Sun, its nucleus is not warm enough to support much water-ice sublimation, the main source of activity for most comets, which usually becomes dominant when within $\sim 3$ au of the Sun. Thus, 29P/SW1's coma must be generated by a different mechanism, as with other distantly active comets (Womack et al. 2017). Many alternatives have been considered for distant activity, including sublimation of a cosmogonically abundant low-condensation temperature ice, an amorphous-tocrystalline phase change of water-ice, HCN polymerization, cryovolcanism, or meteoroid impacts (Prialnik \& Bar-Nun 1990; Senay \& Jewitt 1994; Enzian et al. 1997; Gronkowski 2004; Miles 2016; Schambeau et al. 2019).

A strong candidate for involvement with the activity is $\mathrm{CO}$ outgassing, since its emission was detected in 29P/SW1 over $25 \mathrm{yr}$ ago and has been measured on numerous occasions at millimeter and infrared wavelengths with high enough production rates to support lift off of the observed dust coma (Senay \& Jewitt 1994; Crovisier et al. 1995; Gunnarsson et al. 2008; Paganini et al. 2013). In fact, $\mathrm{CO}$ has the highest production rate by far of all molecules measured in 29P/SW1. In Table 2, we list the measured production rates (or lowest significant limit obtained) for volatile species from the literature and then converted these to equivalent mass-loss rates, which we calculated using appropriate atomic mass units. The range of dust mass-loss rates found in the literature is also given. Besides $\mathrm{CO}$, other volatiles present in the coma are $\mathrm{H}_{2} \mathrm{O}$ (Ootsubo et al. 2012), CN (Cochran \& Cochran 1991), $\mathrm{CO}^{+}$(Cochran et al. 1980), and $\mathrm{N}_{2}^{+}$(Korsun et al. 2008; Ivanova et al. 2016). Water vapor was detected with production rates $\sim 20 \%$ of typical CO (Ootsubo et al. 2012) and there is a preliminary report that
Table 2

Measured and Derived Properties of Dust and Gas in 29P/SW1 Coma

\begin{tabular}{lcccc}
\hline \hline Species & $\begin{array}{c}\text { Production } \\
\text { Rate } \\
\left(10^{27} \mathrm{~mol} \mathrm{~s}^{-1}\right)\end{array}$ & $\begin{array}{c}\text { Mass-loss } \\
\text { Rate }^{\mathrm{a}} \\
\left(\mathrm{kg} \mathrm{s}^{-1}\right)\end{array}$ & $\begin{array}{c}\text { Expansion } \\
\text { Speed } \\
\left(\mathrm{km} \mathrm{s}^{-1}\right)\end{array}$ & Reference \\
\hline Dust & $\ldots$ & $430-4700$ & $0.05-0.15$ & $(1),(2),(3)$, \\
& & & & $(4),(5)$, \\
$\mathrm{CO}$ & $10-70$ & $460-3200$ & $0.20-0.50$ & $(7),(14)$ \\
$\mathrm{H}_{2} \mathrm{O}$ & 6.3 & 188 & $\ldots$ & $(13)$ \\
$\mathrm{N}_{2}\left(\mathrm{~N}_{2}^{+}\right)^{\mathrm{b}}$ & 0.39 & 17 & $\ldots$ & $(7),(9)$ \\
$\mathrm{HCN}(\mathrm{CN})^{\mathrm{c}}$ & 0.008 & 0.3 & $\ldots$ & $(10)$ \\
$\mathrm{H}_{2} \mathrm{CO}$ & 0.1 & $<5$ & $\ldots$ & $(12)$ \\
$\mathrm{CS}$ & $<0.21$ & $<15$ & $\ldots$ & $(12)$ \\
$\mathrm{CO}_{2}$ & $<0.35$ & $<25$ & $\ldots$ & $(8)$ \\
$\mathrm{C}_{2} \mathrm{H}_{6}$ & $<0.57$ & $<28$ & $\ldots$ & $(11)$ \\
$\mathrm{CH}_{3} \mathrm{OH}$ & $<0.55$ & $<29$ & $\ldots$ & $(12)$ \\
$\mathrm{CH}_{4}$ & $<1.3$ & $<34$ & $\ldots$ & $(11)$ \\
$\mathrm{H}_{2} \mathrm{~S}$ & $<1$ & $<56$ & $\ldots$ & $(12)$ \\
$\mathrm{HC}_{3} \mathrm{~N}$ & $<0.95$ & $<81$ & $\ldots$ & $(11)$ \\
$\mathrm{C}_{2} \mathrm{H}_{2}$ & $<2.7$ & $<117$ & $\ldots$ & $(11)$ \\
$\mathrm{NH}_{3}$ & $<10.9$ & $<309$ & $\ldots$ & \\
\hline
\end{tabular}

Notes.

${ }^{a}$ Gaseous mass-loss rates were calculated using production rates and appropriate atomic mass units.

${ }^{b}$ The $\mathrm{N}_{2}$ production rate is inferred from $\mathrm{CO}^{+}, \mathrm{N}_{2}^{+}$, and $\mathrm{CO}$ measurements (Womack et al. 2017).

c The $\mathrm{HCN}$ production rate is inferred from $Q(\mathrm{CN})$.

References. (1) Fulle (1992), (2) Ivanova et al. (2011), (3) Shi et al. (2014), (4) Gunnarsson et al. (2002), (5) Fulle et al. (1998), (6) Feldman et al. (1996), (7) Womack et al. (2017), (8) Ootsubo et al. (2012), (9) Ivanova et al. (2016), (10) Cochran \& Cochran (1991), (11) Paganini et al. (2013), (12) Biver (1997), (13) Biver et al. (1999), (14) Schambeau (2018).

high-resolution spectra of $\mathrm{H}_{2} \mathrm{O}$ indicates that the emission is consistent with sublimation from icy grains in the coma (Bockelee-Morvan et al. 2014). CN is a short-lived radical daughter species, probably created from photodestruction of $\mathrm{HCN}$ in the coma. HCN emission was claimed to be detected in $29 \mathrm{P} / \mathrm{SW} 1$ with relative abundances similar to that of Hale-Bopp at $6 \mathrm{au}$, and also with a spectral line profile consistent with sublimating from icy grains in the coma; however, no quantitative values have been published yet (Bockelee-Morvan et al. 2014). Thus, the HCN values listed in Table 2 were derived from CN measurement-see Womack et al. (2017) for more details. The amount of $\mathrm{CO}^{+}$detected during outbursting (Cochran et al. 1980) and quiescent stages (Larson 1980) is consistent with forming from $\mathrm{CO}$ and not $\mathrm{CO}_{2}$ (Ivanova et al. 2019). $\mathrm{N}_{2}^{+}$is detected in very small amounts, which was interpreted as evidence for $\mathrm{N}_{2}$ being present only in small amounts relative to $\mathrm{CO}$ in the coma (Womack et al. 2017). Significant upper limits were obtained for the highly volatile $\mathrm{CO}_{2}$ (Ootsubo et al. 2012), and $\mathrm{C}_{2} \mathrm{H}_{6}, \mathrm{CH}_{4}, \mathrm{C}_{2} \mathrm{H}_{2}, \mathrm{NH}_{3}$, and $\mathrm{CH}_{3} \mathrm{OH}$ (Paganini et al. 2013), which indicate that all of these make much less of a contribution to the gas coma than $\mathrm{CO}$ (even when combined, see Table 2).

With a perihelion distance of only $q=5.76 \mathrm{au}$, the continuous presence of both dust and CO comae makes 29P/ SW1 unique among the comet and Centaur populations. This may be typical of the beginning state of cometary activity for 
Centaurs as they progress toward a JFC orbit (Sarid et al. 2019).

There are many studies documenting 29P/SW1 quiescent and outbursting dust activity (Trigo-Rodríguez et al. 2008; Hosek et al. 2013; Miles et al. 2016; Schambeau et al. 2019), but very few include both dust and gas components measured at or about the same time. Analysis of optical spectra of 29P/ SW1 obtained in 1990 indicated that the $\mathrm{CO}^{+}$and dust production were not strongly coupled during an outburst. The column density of the (3-0) $\mathrm{CO}^{+}$band at $\sim 4000 \AA$ was observed to increase by a factor of 2.5 , while the continuum magnitude (dust), which was measured simultaneously at $\sim 4450 \AA$ was unchanged. One interpretation was that this was evidence that the gas and dust were not entrained (Cochran et al. 1991), but this depended on an interpretation that $\mathrm{CO}^{+}$ was a good tracer of the $\mathrm{CO}$ production rate. In comets, $\mathrm{CO}^{+}$ can be produced by the photoionization of $\mathrm{CO}$, but this process is slow and scales as $r^{-2}$, and is therefore ineffective at this large distance from the Sun and is unlikely to be the source of most of the observed $\mathrm{CO}^{+}$(Cochran \& Cochran 1991). Instead, $\mathrm{CO}^{+}$column density and variability can be better explained by solar-wind proton impact onto cometary $\mathrm{CO}$, which is strongly dependent on solar-wind particle velocities (Cochran et al. 1991; Jockers et al. 1992; Ivanova et al. 2019). Thus it appears that for $29 \mathrm{P} / \mathrm{SW} 1, \mathrm{CO}^{+}$variation is not a straightforward proxy for $\mathrm{CO}$ behavior, and is a better tracer of solar-wind particles at 29P/SW1's location for a given date. Thus, the reported variations of $\mathrm{CO}^{+} /$dust continuum during outburst are likely not indicative of real changes of the gas/dust ratio without taking into account contributions from the solar wind.

Another study claimed a lack of correlation between the gas and dust production in $29 \mathrm{P} / \mathrm{SW} 1$ evident in $Q(\mathrm{CO})$ and visible magnitudes (Biver 2001). This conclusion is in contrast to a strong correlation that the author found for long-period comets C/1995 O1 (Hale-Bopp) and C/1997 J2 (Meunier-Dupouy). However, it appears that the study for 29P/SW1 included all $Q$ (CO) and magnitude values, regardless of whether they were obtained during outbursting or quiescent stage. If the Centaur were having either a $\mathrm{CO}$ or dust outburst during any the Biver (2001) measurements, then this would significantly obscure any possible correlation. Comets Hale-Bopp and MeunierDupouy had far fewer outbursts, and so their data were highly likely obtained during when the comets were in a quiescent stage. To test for a correlation between the quiescent gas and dust production rate in this famously outbursting Centaur, one must compare known quiescent gas with quiescent dust production rates. Thus, although useful for showing that 29P/SW1 is a dust-poor comet compared to Hale-Bopp and Meunier-Dupouy, the results from Biver (2001) may not be sufficient to test the hypothesis of whether $\mathrm{CO}$ outgassing is correlated to the dust activity for either the quiescent or the outbursting stages. To address this, the $Q(\mathrm{CO})$ and magnitude values should be simultaneous and they should be taken when $29 \mathrm{P} / \mathrm{SW} 1$ is in a quiescent or outbursting stage for both the gas and dust.

There are also remarkably few detailed models attempting to describe the observational record (Enzian et al. 1997; Kossacki \& Szutowicz 2013; Wesołowski \& Gronkowski 2018). Important observational constraints for testing models of 29P/SW1's behavior are missing: quantifying whether $\mathrm{CO}$ production is connected to dust outbursts, and if so, by how much. In order to assess this, we compiled secular lightcurves derived from visual brightness measurements obtained nearly at the same time as the CO millimeter-wavelength spectra and searched for correlations. In particular, it is widely assumed that $\mathrm{CO}$ outgassing is largely correlated with the dust outbursts in 29P/ SW1, and we decided to test this hypothesis.

\section{Observations}

\subsection{Millimeter-wavelength Spectra}

We monitored emission from the $\mathrm{CO} J=2-1$ transition at $230.53799 \mathrm{GHz}$ toward $29 \mathrm{P} / \mathrm{SW} 1$ with the Arizona Radio Observatory (ARO) $10 \mathrm{~m}$ Submillimeter Telescope (SMT) during 2016 February-May and 2018 November-2019 January (see Table 3). The times listed in the table are at the end of the observing period for each day.

In both observing campaigns, the data were taken with the SMT $1.3 \mathrm{~mm}$ receiver with Atacama Large Millimeter/ submillimeter Array (ALMA) Band 6 sideband-separating mixers in dual polarization. The backend used for the observations consisted of a 500 channel $(2 \times 250)$ filterbank with a resolution of $250 \mathrm{kHz} /$ channel in parallel mode. This frequency resolution corresponds to $0.325 \mathrm{~km} \mathrm{~s}^{-1}$ per channel at the CO (2-1) frequency. The SMT beam diameter at this frequency was $\theta_{B}=32^{\prime \prime}$. All of the scans were taken in the beam-switching position mode with a throw of $+2^{\prime}$ in azimuth, and the system temperatures remained between 200 and $400 \mathrm{~K}$. All the scans were obtained by integrating three minutes on the source and three minutes on the sky for subtraction.

The position of the comet was checked periodically against the ephemeris position provided by JPL Horizons. Tracking was found to be within $<1^{\prime \prime}$ rms during both of the observing epochs. Additionally, pointing and focus was updated on planets and a bright radio-source every 6 to 10 scans.

\subsection{Visible Magnitudes}

We used reports of 29P/SW1's apparent visual magnitude, $m_{v}$, to construct a secular lightcurve spanning the two time periods in which we monitored $Q(\mathrm{CO})$. In order to minimize observer differences, we used magnitudes from experienced observers who were well versed in how to obtain, reduce, and report CCD magnitudes of comets (Larson et al. 1991; M. Womack et al. 2020, in preparation). The data were obtained from the Lesia database of cometary observations ${ }^{3}$ and the Minor Planet Center (MPC) Observation Database ${ }^{4}$ recorded with unfiltered CCDs (Table 4). The magnitudes from the Lesia database of cometary observations were obtained with telescopes ranging between $0.2 \mathrm{~m}$ and $0.4 \mathrm{~m}$ in aperture diameter by the observers J.-F. Soulier, F. Kugel, J.-G.Bosch, J. Nicolas, T. Noel, and P. Ditz. The observations from the MPC were obtained by the TRAnsiting Planets and Planetesimals Small Telescope-South (TRAPPIST; $0.6 \mathrm{~m}$ telescope in La Silla, Chile) and by J. Drummond (0.35 $\mathrm{m}$ telescope at Possum Observatory in Gisborne, NZ).

We chose nuclear magnitudes measured with photometric apertures of $5^{\prime \prime}-7$ " centered on the nucleus, in contrast to the much brighter total magnitudes, which generally encompass most of the visible coma. Coma contamination typically occurs close to the nucleus; however, nuclear magnitudes are more

\footnotetext{
3 http://lesia.obspm.fr/comets/index.php

4 https://minorplanetcenter.net/db_search
} 
Table 3

CO $J=2-1$ Observations of 29P/SW1 Using the ARO SMT $10 \mathrm{~m}$ Telescope

\begin{tabular}{|c|c|c|c|c|c|c|}
\hline UT Date & $\begin{array}{c}r \\
\text { (au) }\end{array}$ & $\begin{array}{c}\Delta \\
(\mathrm{au})\end{array}$ & $\begin{array}{c}T_{A}^{*} d v \\
\left(\mathrm{mK} \mathrm{km} \mathrm{s}^{-1}\right)\end{array}$ & $\begin{array}{l}\Delta v_{\mathrm{FWHM}} \\
\left(\mathrm{km} \mathrm{s}^{-1}\right)\end{array}$ & $\begin{array}{c}\delta v \\
\left(\mathrm{~km} \mathrm{~s}^{-1}\right)\end{array}$ & $\begin{array}{c}Q \times 10^{28} \\
\left(\mathrm{~mol} \mathrm{~s}^{-1}\right)\end{array}$ \\
\hline 2016 Feb 25.7 & 5.96 & 6.59 & $48 \pm 3$ & 0.72 & -0.32 & ${ }^{*} 3.00 \pm 0.17$ \\
\hline 2016 Feb 26.7 & 5.96 & 6.57 & $59 \pm 5$ & 0.89 & -0.39 & $3.67 \pm 0.28$ \\
\hline 2016 Feb 29.7 & 5.96 & 6.54 & $55 \pm 5$ & 0.74 & -0.48 & $3.38 \pm 0.28$ \\
\hline 2016 Mar 1.8 & 5.96 & 6.52 & $49 \pm 3$ & 0.83 & -0.36 & ${ }^{*} 3.05 \pm 0.20$ \\
\hline 2016 Mar 3.8 & 5.96 & 6.50 & $48 \pm 3$ & 0.92 & -0.36 & ${ }^{*} 3.01 \pm 0.20$ \\
\hline 2016 Mar 31.6 & 5.95 & 6.09 & $59 \pm 5$ & 0.68 & -0.38 & $3.62 \pm 0.28$ \\
\hline 2016 Apr 9.6 & 5.95 & 5.94 & $65 \pm 3$ & 0.80 & -0.33 & $4.01 \pm 0.16$ \\
\hline 2016 Apr 15.6 & 5.95 & 5.85 & $42 \pm 3$ & 0.70 & -0.29 & ${ }^{*} 2.62 \pm 0.18$ \\
\hline 2016 Apr 24.6 & 5.94 & 5.70 & $54 \pm 3$ & 1.06 & -0.25 & $3.34 \pm 0.15$ \\
\hline 2016 May 29.5 & 5.93 & 5.20 & $59 \pm 4$ & 0.74 & -0.34 & $3.63 \pm 0.18$ \\
\hline 2018 Nov 2.2 & 5.76 & 5.17 & $89 \pm 9$ & 0.99 & -0.14 & ${ }^{*} 4.16 \pm 0.43$ \\
\hline 2018 Dec 21.1 & 5.76 & 5.92 & $42 \pm 2$ & 0.52 & -0.43 & ${ }^{*} 2.23 \pm 0.13$ \\
\hline 2019 Jan 8.1 & 5.76 & 6.19 & $79 \pm 3$ & 0.85 & -0.34 & ${ }^{*} 4.40 \pm 0.17$ \\
\hline 2019 Jan 15.0 & 5.76 & 6.29 & $57 \pm 4$ & 0.56 & -0.36 & ${ }^{*} 3.26 \pm 0.21$ \\
\hline
\end{tabular}

Note. ${ }^{*} Q(\mathrm{CO})$ values marked with an asterisk are considered quiescent. (see Section 3).

likely than total magnitudes to pick up short-term changes in cometary activity.

We corrected the apparent visual magnitudes, $m_{v}$, for the Centaur's geocentric distance according to

$$
m(1, r, \theta)=m_{v}-5 \log _{10}(\Delta),
$$

where $\Delta$ is the comet's geocentric distance and ranges from 5.2 to $6.6 \mathrm{au}$ for the observing periods, and $m(1, r, \theta)$ is often referred to as the heliocentric magnitude, or $m_{\text {helio }}$. We also applied a phase correction to account for scattered light using the phase function $\phi[\theta]$ normalized to $0^{\circ}$ with

$$
m(1, r, 0)=m(1, r, \theta)+2.5 \log (\phi[\theta]),
$$

following the method of Schleicher \& Bair (2011). ${ }^{5}$

\section{Results}

The CO millimeter-wavelength spectra and visible magnitudes presented here are similar to data reported elsewhere for the Centaur during its quiescent and outbursting stages (Senay \& Jewitt 1994; Crovisier et al. 1995; Cabot et al. 1996; Biver 1997; Festou et al. 2001; Gunnarsson et al. 2003, 2008; Trigo-Rodríguez et al. 2008; Miles et al. 2016).

Emission from the $\mathrm{CO}(2-1)$ rotational transition was readily detected in individual six-minute scans, and these were co-added to produce one spectrum for each day (see Figure 1). Using a Gaussian fit to the co-added spectrum the average fullwidth half-maximum line width for the $\mathrm{CO}$ emission was $\Delta V_{\mathrm{FWHM}}=0.87 \pm 0.33 \mathrm{~km} \mathrm{~s}^{-1}$. Within the uncertainties, this is consistent with what is measured with other telescopes (Senay \& Jewitt 1994; Gunnarsson et al. 2003), considering that we did not fully resolve the line. The emission was blueshifted by an average of $\delta v=-0.32 \pm 0.16 \mathrm{~km} \mathrm{~s}^{-1}$, in

\footnotetext{
5 https://asteroid.lowell.edu/comet/dustphaseHM_table.html
}

agreement with what is typically observed for $\mathrm{CO}$ emission in cometary objects beyond 5 au (Womack et al. 2017). In order to derive $\mathrm{CO}$ column densities from the spectra, we used measured fluxes of the $2-1$ line, and an excitation model that assumes fluorescence and collisional excitation following the modeling efforts of Crovisier \& Le Bourlot (1983) and Biver (1997). We assumed an optically thin gas, excitation and rotational temperatures of $10 \mathrm{~K}$, and a gas expansion velocity of $0.3 \mathrm{~km} \mathrm{~s}^{-1}$. CO production rates were calculated from the column densities using a simple isotropic and constant radial outgassing model (Haser 1957). Line profile measurements and derived production rates are listed in Table 3.

Throughout our 21 days of CO observations, 29P/SW1 always produced at least $Q(\mathrm{CO})=2-3 \times 10^{28} \mathrm{~mol} \mathrm{~s}^{-1}$ with a few variations up to $\sim 4 \times 10^{28} \mathrm{~mol} \mathrm{~s}^{-1}$ and a peak of $Q(\mathrm{CO})$ $\sim 6 \times 10^{28} \mathrm{~mol} \mathrm{~s}^{-1}$ (see Table 3 and Figure 2). The corrected visible magnitudes ranged from $m(1, r, 0)=9.3$ to 13.7 .

We calculated the average quiescent $\mathrm{CO}$ production rate and average quiescent heliocentric magnitude for each observing period using the data in Tables 3 and 4. For the 2016 epoch we define the quiescent $\mathrm{CO}$ stage as the apparent baseline level of the $\mathrm{CO}$ production rate surrounding the $\mathrm{CO}$ outburst, that is values of $Q(\mathrm{CO})<3.2 \times 10^{28} \mathrm{~mol} \mathrm{~s}^{-1}$. We did not include elevated $\mathrm{CO}$ values that appeared to coincide with increased dust production. With respect to 2018-2019, we did not definitely detect a compelling coincidence of elevated $\mathrm{CO}$ and dust production, so all measurements were used to determine the quiescent average value in this epoch. Quiescent values of $Q(\mathrm{CO})$ are marked with an asterisk in Table 3.

For 2016 the average quiescent $\mathrm{CO}$ production rate is $Q(\mathrm{CO})=(2.9 \pm 0.2) \times 10^{28} \mathrm{~mol} \mathrm{~s}^{-1}$ and the average quiescent heliocentric magnitude is $m(1, r, 0)=12.9 \pm 0.2$. For the 2018-2019 data the average quiescent values are $Q(\mathrm{CO})=$ $(3.6 \pm 0.7) \times 10^{28} \mathrm{~mol} \mathrm{~s}^{-1}$ and $m(1, r, 0)=12.5 \pm 0.2$. The uncertainties given in the averages are one standard deviation. 
Table 4

Nuclear Magnitudes, $m_{v}$, of 29P/SW1 Obtained from the Minor Planet Center and the Lesia Database of Cometary Observations for Both Observing Epochs

\begin{tabular}{|c|c|c|c|c|}
\hline UT Date & $\Delta(\mathrm{au})$ & $m_{v}$ & $m(1, r, 0)$ & $D(m)$ \\
\hline 2016 Feb 20.7 & 6.65 & 16.8 & 12.4 & 0.35 \\
\hline 2016 Feb 24.4 & 6.63 & 17.2 & 12.8 & 0.60 \\
\hline 2016 Mar 3.3 & 6.59 & 17.3 & 12.9 & 0.40 \\
\hline 2016 Mar 8.3 & 6.51 & 17.3 & 12.9 & 0.40 \\
\hline 2016 Mar 9.3 & 6.44 & 17.2 & 12.8 & 0.40 \\
\hline 2016 Mar 10.3 & 6.43 & 17.3 & 12.9 & 0.40 \\
\hline 2016 Mar 11.3 & 6.40 & 17.3 & 12.9 & 0.40 \\
\hline 2016 Mar 12.3 & 6.39 & 17.4 & 13.0 & 0.40 \\
\hline 2016 Mar 14.3 & 6.36 & 14.8 & 10.4 & 0.40 \\
\hline 2016 Mar 16.3 & 6.33 & 15.0 & 10.6 & 0.40 \\
\hline 2016 Mar 17.3 & 6.31 & 15.1 & 10.7 & 0.40 \\
\hline 2016 Mar 18.3 & 6.30 & 15.3 & 10.9 & 0.40 \\
\hline 2016 Mar 19.3 & 6.28 & 15.1 & 10.7 & 0.40 \\
\hline 2016 Mar 21.3 & 6.25 & 15.7 & 11.3 & 0.40 \\
\hline 2016 Mar 30.3 & 6.12 & 16.6 & 12.3 & 0.35 \\
\hline 2016 Apr 4.3 & 6.04 & 16.6 & 12.3 & 0.32 \\
\hline 2016 Apr 10.3 & 5.94 & 17.0 & 12.7 & 0.40 \\
\hline 2016 Apr 12.3 & 5.91 & 17.1 & 12.8 & 0.40 \\
\hline 2016 Apr 13.3 & 5.89 & 17.1 & 12.9 & 0.40 \\
\hline 2016 Apr 14.3 & 5.88 & 17.1 & 12.9 & 0.40 \\
\hline 2016 Apr 16.3 & 5.84 & 17.3 & 13.1 & 0.40 \\
\hline 2016 Apr 19.1 & 5.79 & 17.4 & 13.2 & 0.30 \\
\hline 2016 Apr 22.3 & 5.75 & 17.2 & 13.0 & 0.40 \\
\hline 2016 Apr 23.3 & 5.73 & 16.9 & 12.7 & 0.40 \\
\hline 2016 Apr 24.1 & 5.71 & 16.8 & 12.6 & 0.30 \\
\hline 2016 Apr 25.3 & 5.70 & 17.3 & 13.1 & 0.40 \\
\hline 2016 Apr 26.3 & 5.68 & 17.0 & 12.8 & 0.40 \\
\hline 2016 Apr 27.1 & 5.67 & 17.4 & 13.2 & 0.40 \\
\hline 2016 May 14.0 & 5.41 & 17.7 & 13.7 & 0.40 \\
\hline 2016 May 17.0 & 5.36 & 17.4 & 13.4 & 0.20 \\
\hline 2016 May 18.0 & 5.35 & 17.0 & 13.0 & 0.20 \\
\hline 2016 May 20.0 & 5.32 & 16.7 & 12.7 & 0.20 \\
\hline 2016 May 21.0 & 5.31 & 17.0 & 13.0 & 0.20 \\
\hline 2016 May 22.0 & 5.30 & 16.8 & 12.9 & 0.20 \\
\hline 2016 May 23.0 & 5.28 & 16.7 & 12.8 & 0.20 \\
\hline 2016 May 24.0 & 5.27 & 16.9 & 13.0 & 0.20 \\
\hline 2016 May 26.0 & 5.24 & 16.8 & 12.9 & 0.20 \\
\hline 2016 May 28.0 & 5.22 & 16.1 & 12.2 & 0.20 \\
\hline 2016 May 30.0 & 5.20 & 16.6 & 12.7 & 0.20 \\
\hline 2016 May 31.0 & 5.18 & 16.6 & 12.7 & 0.20 \\
\hline 2016 Jun 2.0 & 5.16 & 16.5 & 12.6 & 0.20 \\
\hline 2016 Jun 3.0 & 5.15 & 16.7 & 12.9 & 0.20 \\
\hline 2016 Jun 4.3 & 5.14 & 16.6 & 12.8 & 0.35 \\
\hline 2016 Jun 5.0 & 5.13 & 16.5 & 12.7 & 0.20 \\
\hline 2016 Jun 5.3 & 5.13 & 16.5 & 12.7 & 0.35 \\
\hline 2016 Jun 6.0 & 5.12 & 16.3 & 12.5 & 0.20 \\
\hline 2018 Nov 1.9 & 5.15 & 16.2 & 12.3 & 0.40 \\
\hline 2018 Nov 2.7 & 5.17 & 16.1 & 12.2 & 0.30 \\
\hline 2018 Nov 3.9 & 5.18 & 16.5 & 12.6 & 0.20 \\
\hline 2018 Nov 4.9 & 5.19 & 16.4 & 12.5 & 0.40 \\
\hline 2018 Nov 6.1 & 5.22 & 16.4 & 12.4 & 0.40 \\
\hline 2018 Nov 8.9 & 5.25 & 16.6 & 12.6 & 0.40 \\
\hline 2018 Nov 9.9 & 5.26 & 16.6 & 12.6 & 0.40 \\
\hline 2018 Nov 10.9 & 5.28 & 16.3 & 12.3 & 0.40 \\
\hline 2018 Nov 11.9 & 5.29 & 16.4 & 12.4 & 0.40 \\
\hline 2018 Nov 13.0 & 5.32 & 16.4 & 12.4 & 0.40 \\
\hline 2018 Nov 13.7 & 5.32 & 16.4 & 12.5 & 0.20 \\
\hline 2018 Nov 13.9 & 5.32 & 16.6 & 12.6 & 0.40 \\
\hline 2018 Nov 14.9 & 5.34 & 16.5 & 12.5 & 0.40 \\
\hline 2018 Nov 15.7 & 5.35 & 16.1 & 12.1 & 0.20 \\
\hline 2018 Nov 15.9 & 5.35 & 16.5 & 12.5 & 0.40 \\
\hline 2018 Nov 16.8 & 5.37 & 16.2 & 12.2 & 0.30 \\
\hline 2018 Nov 17.7 & 5.38 & 16.5 & 12.5 & 0.30 \\
\hline
\end{tabular}

Table 4

(Continued)

\begin{tabular}{|c|c|c|c|c|}
\hline UT Date & $\Delta(\mathrm{au})$ & $m_{v}$ & $m(1, r, 0)$ & $D(m)$ \\
\hline 2018 Nov 18.7 & 5.40 & 16.5 & 12.4 & 0.30 \\
\hline 2018 Nov 19.9 & 5.41 & 16.6 & 12.5 & 0.40 \\
\hline 2018 Nov 20.9 & 5.43 & 16.7 & 12.6 & 0.40 \\
\hline 2018 Nov 21.9 & 5.45 & 13.4 & 9.3 & 0.40 \\
\hline 2018 Nov 22.7 & 5.46 & 13.6 & 9.5 & 0.20 \\
\hline 2018 Nov 22.9 & 5.46 & 13.4 & 9.3 & 0.40 \\
\hline 2018 Nov 23.9 & 5.48 & 13.5 & 9.4 & 0.40 \\
\hline 2018 Nov 24.7 & 5.49 & 13.9 & 9.8 & 0.20 \\
\hline 2018 Nov 24.9 & 5.49 & 13.9 & 9.8 & 0.40 \\
\hline 2018 Nov 25.9 & 5.51 & 14.3 & 10.2 & 0.40 \\
\hline 2018 Nov 27.0 & 5.54 & 14.7 & 10.6 & 0.40 \\
\hline 2018 Nov 27.7 & 5.54 & 14.6 & 10.5 & 0.20 \\
\hline 2018 Nov 28.0 & 5.56 & 15.0 & 10.9 & 0.40 \\
\hline 2018 Nov 28.7 & 5.56 & 14.9 & 10.8 & 0.20 \\
\hline 2018 Nov 28.8 & 5.56 & 15.9 & 11.7 & 0.41 \\
\hline 2018 Nov 29.7 & 5.57 & 15.6 & 11.5 & 0.20 \\
\hline 2018 Nov 30.0 & 5.59 & 15.7 & 11.6 & 0.40 \\
\hline 2018 Dec 3.7 & 5.64 & 16.0 & 11.8 & 0.20 \\
\hline 2018 Dec 4.0 & 5.65 & 16.2 & 12.0 & 0.40 \\
\hline 2018 Dec 7.0 & 5.71 & 16.5 & 12.3 & 0.40 \\
\hline 2018 Dec 8.0 & 5.72 & 16.3 & 12.2 & 0.40 \\
\hline 2018 Dec 9.0 & 5.73 & 14.6 & 10.4 & 0.40 \\
\hline 2018 Dec 10.0 & 5.75 & 14.8 & 10.6 & 0.40 \\
\hline 2018 Dec 10.7 & 5.75 & 14.9 & 10.6 & 0.20 \\
\hline 2018 Dec 11.7 & 5.76 & 15.2 & 11.0 & 0.20 \\
\hline 2018 Dec 11.8 & 5.76 & 16.1 & 11.9 & 0.41 \\
\hline 2018 Dec 13.0 & 5.80 & 15.7 & 11.5 & 0.40 \\
\hline 2018 Dec 13.7 & 5.80 & 16.1 & 11.9 & 0.30 \\
\hline 2018 Dec 14.8 & 5.81 & 16.2 & 12.0 & 0.20 \\
\hline 2018 Dec 16.0 & 5.85 & 16.1 & 11.9 & 0.40 \\
\hline 2018 Dec 17.7 & 5.87 & 16.4 & 12.2 & 0.20 \\
\hline 2018 Dec 19.7 & 5.89 & 16.6 & 12.4 & 0.20 \\
\hline 2018 Dec 20.7 & 5.91 & 16.8 & 12.6 & 0.20 \\
\hline 2018 Dec 22.0 & 5.94 & 16.6 & 12.3 & 0.40 \\
\hline 2018 Dec 23.0 & 5.95 & 17.1 & 12.8 & 0.40 \\
\hline 2018 Dec 23.7 & 5.96 & 16.9 & 12.6 & 0.20 \\
\hline 2018 Dec 24.0 & 5.97 & 16.7 & 12.4 & 0.40 \\
\hline 2018 Dec 24.7 & 5.97 & 16.9 & 12.6 & 0.20 \\
\hline 2018 Dec 25.7 & 5.99 & 16.9 & 12.6 & 0.20 \\
\hline 2018 Dec 25.7 & 5.99 & 17.4 & 13.1 & 0.41 \\
\hline 2018 Dec 26.0 & 6.01 & 17.0 & 12.7 & 0.40 \\
\hline 2018 Dec 26.7 & 6.01 & 16.9 & 12.6 & 0.20 \\
\hline 2018 Dec 26.7 & 6.01 & 17.3 & 13.0 & 0.20 \\
\hline 2018 Dec 27.7 & 6.02 & 16.7 & 12.4 & 0.20 \\
\hline 2018 Dec 28.7 & 6.04 & 16.5 & 12.2 & 0.20 \\
\hline 2018 Dec 30.7 & 6.07 & 16.5 & 12.2 & 0.20 \\
\hline 2018 Dec 31.7 & 6.08 & 16.6 & 12.3 & 0.20 \\
\hline 2019 Jan 1.7 & 6.09 & 16.4 & 12.1 & 0.20 \\
\hline 2019 Jan 2.7 & 6.11 & 16.3 & 12.0 & 0.20 \\
\hline 2019 Jan 2.7 & 6.11 & 16.7 & 12.4 & 0.20 \\
\hline 2019 Jan 3.7 & 6.12 & 16.0 & 11.7 & 0.20 \\
\hline 2019 Jan 5.0 & 6.15 & 15.8 & 11.5 & 0.40 \\
\hline 2019 Jan 6.0 & 6.16 & 15.8 & 11.5 & 0.40 \\
\hline 2019 Jan 6.7 & 6.16 & 16.0 & 11.7 & 0.20 \\
\hline 2019 Jan 7.7 & 6.18 & 15.9 & 11.6 & 0.20 \\
\hline 2019 Jan 8.7 & 6.19 & 16.1 & 11.8 & 0.20 \\
\hline 2019 Jan 9.0 & 6.21 & 16.3 & 12.0 & 0.40 \\
\hline 2019 Jan 10.0 & 6.22 & 16.3 & 12.0 & 0.40 \\
\hline 2019 Jan 10.7 & 6.22 & 16.3 & 12.0 & 0.20 \\
\hline 2019 Jan 11.7 & 6.23 & 16.5 & 12.2 & 0.20 \\
\hline 2019 Jan 12.7 & 6.25 & 16.7 & 12.4 & 0.20 \\
\hline 2019 Jan 13.7 & 6.26 & 16.4 & 12.1 & 0.20 \\
\hline 2019 Jan 14.7 & 6.27 & 16.8 & 12.5 & 0.20 \\
\hline 2019 Jan 15.7 & 6.29 & 16.7 & 12.4 & 0.20 \\
\hline
\end{tabular}


Table 4

(Continued)

\begin{tabular}{lcccc}
\hline \hline UT Date & $\Delta(\mathrm{au})$ & $m_{v}$ & $m(1, r, 0)$ & $D(m)$ \\
\hline 2019 Jan 21.7 & 6.36 & 16.8 & 12.5 & 0.20 \\
2019 Jan 23.7 & 6.39 & 16.7 & 12.4 & 0.20 \\
2019 Jan 24.7 & 6.40 & 16.7 & 12.4 & 0.20 \\
2019 Jan 28.7 & 6.44 & 16.6 & 12.3 & 0.20 \\
\hline
\end{tabular}

Note. $D$ is the telescope aperture diameter.

\section{$12 \mathrm{CO}(2-1)$ Spectrum of 29P/Schwassmann-Wachmann}

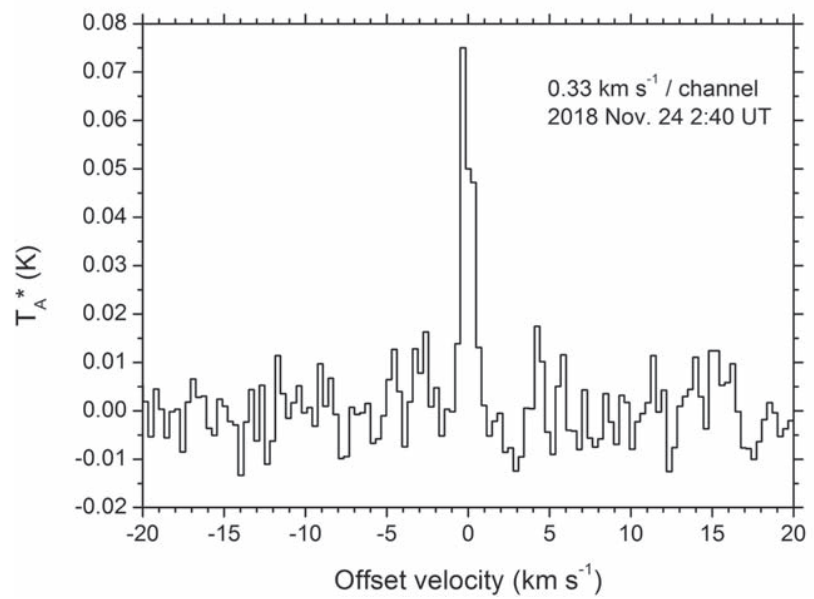

Figure 1. Typical CO spectrum of comet $29 \mathrm{P} / \mathrm{SW} 1$ obtained over $\sim 3 \mathrm{hr}$ with the ARO $10 \mathrm{~m}$ SMT using $250 \mathrm{kHz}\left(0.33 \mathrm{~km} \mathrm{~s}^{-1}\right)$ channel resolution.

In order to test the hypothesis that $\mathrm{CO}$ outbursts were correlated with dust outbursts we plotted the $Q(\mathrm{CO})$ and $m(1, r$, 0 ) values versus Julian date on the same graph (see Figure 2). We aligned the average quiescent heliocentric magnitude and $Q$ (CO) values, which are designated with a horizontal dashed line in Figure 2.

The observations recorded the evolution of one $\mathrm{CO}$ outburst over five days. Starting at 2016 February 25.7 the CO production rate doubled within $70 \mathrm{hr}$ and then returned to the original quiescent production rate approximately three days later. Within the uncertainties, there is no measurable change to the CO line profile during the gas outburst. There are four short-lived dust outbursts of $\Delta m>1 \mathrm{mag}$ in the visible lightcurve, where $\Delta m$ is the change in corrected visible magnitude $m(1, r, 0)$ (Table 4). During the outbursts, the dust coma increased brightness within a few hours and decayed over a few days to weeks.

\section{Discussion}

\subsection{Quiescent Activity of Gas and Dust}

Cometary activity is governed by the structure and composition of the nucleus, including how well the dust, ice, and gas components are integrated (Prialnik et al. 2004). Activity is typically generated by the sublimation of water-ice, which is the largest icy constituent of most nuclei. However, at 29P/SW1's orbital distance water-ice sublimation off the nucleus surface is inefficient and instead, the volatile most likely responsible for driving the observed dust activity is considered to be $\mathrm{CO}$ based on its high production rates which match, and often exceed, the dust mass-loss rate (Senay \& Jewitt 1994).
29P/SW1 gas and dust lightcurves
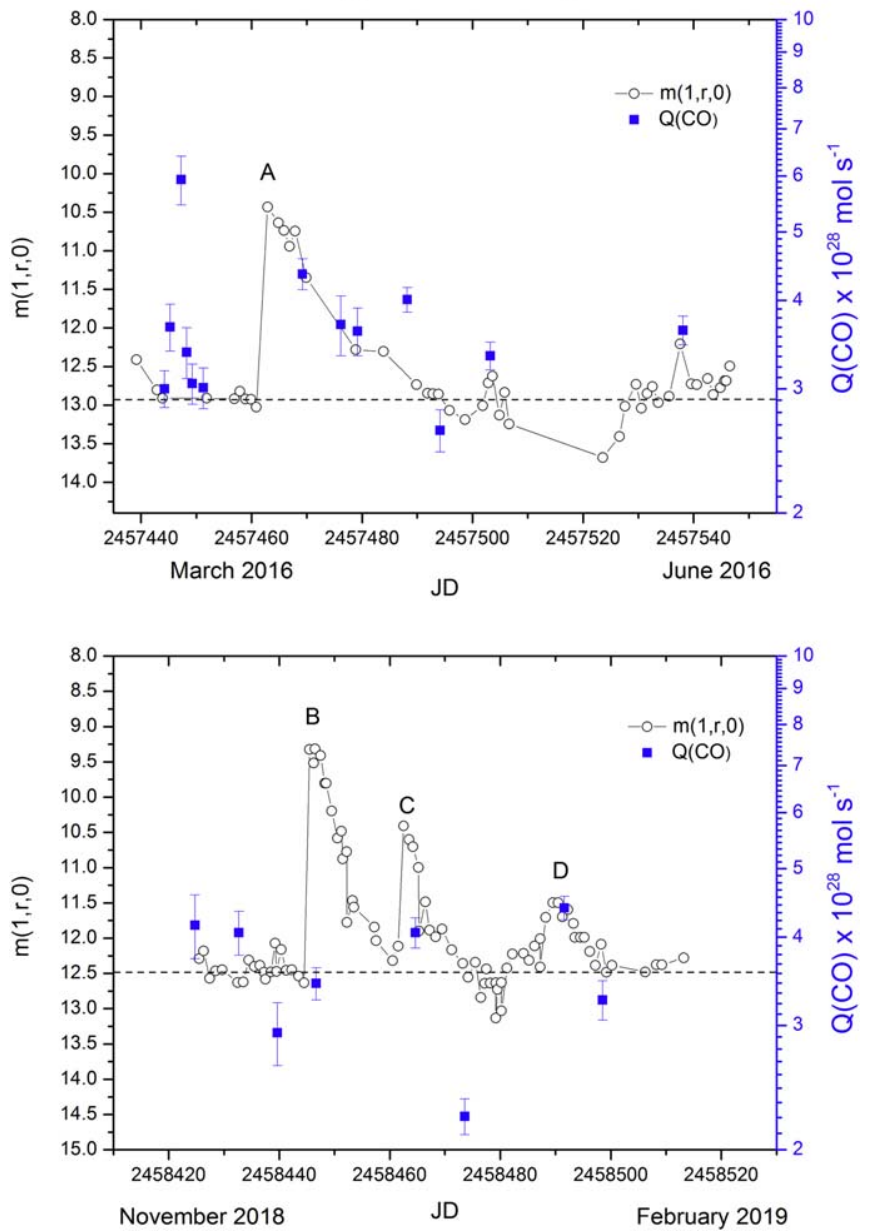

Figure 2. 29P/SW1 lightcurves of CO production rates (blue filled squares) and visible magnitudes corrected for the heliocentric distance and phase angle (open circles) during 2016 (top panel) and 2018-2019 (bottom panel). Each panel spans 120 days. One CO outburst was detected on 2016 February 28, which did not coincide with a dust outburst. Four dust outbursts were seen on 2016 March 14, 2018 November 22, 2018 December 9, and 2019 January 8 (marked as A, B, C, and D respectively). The average quiescent stage values of $m(1, r, 0)$ and $Q(\mathrm{CO})$ are indicated by a horizontal dashed line. The dust coma appeared $\sim 0.4$ mag brighter in 2018-2019 when the Centaur was slightly $(\sim 0.2 \mathrm{au})$ closer to the Sun.

As Table 2 shows, no other volatile measured so far in 29P/ $\mathrm{SW} 1$ competes with $\mathrm{CO}$ in terms of mass-loss rates, including $\mathrm{CO}_{2}, \mathrm{CH}_{4}, \mathrm{H}_{2} \mathrm{CO}, \mathrm{CH}_{3} \mathrm{OH}, \mathrm{N}_{2}$, or $\mathrm{NH}_{3} . \mathrm{O}_{2}$ is another possibly abundant molecule with high volatility $\left(T_{\text {sub }} \sim 24 \mathrm{~K}\right.$; Yamamoto 1985), but it is very difficult to observe with telescopes (LuspayKuti et al. 2018; Taquet et al. 2018) and no measurements of its abundance exist for $29 \mathrm{P} / \mathrm{SW} 1$. Using mass spectroscopy, $\mathrm{O}_{2}$ was detected in the comae of $67 \mathrm{P} /$ Churyomov-Gerasimenko and Halley with amounts of $\sim 4 \%$ relative to water (Bieler et al. 2015; Rubin et al. 2015). If $\mathrm{O}_{2}$ is released with the same percentage to water in $29 \mathrm{P} / \mathrm{SW} 1$ 's coma, then it would be present at $\mathrm{Q}\left(\mathrm{O}_{2}\right) \sim 2.4 \times 10^{26} \mathrm{~mol} \mathrm{~s}^{-1}\left(13 \mathrm{~kg} \mathrm{~s}^{-1}\right)$, placing it well below CO's production rate and on par with what we infer for $\mathrm{N}_{2}$ (see Table 2). Of course, 67P and Halley were closer to the Sun when their measurements were made and they had more water-ice sublimation than 29P/SW1. Given the low sublimation temperature, if $\mathrm{O}_{2}$ is present in $29 \mathrm{P} / \mathrm{SW} 1$ then it may play a small role in the activity, but is not expected to rival CO. After 
considering the evidence for all likely competitors, we affirm that $\mathrm{CO}$ is the volatile most likely to be involved in generating the observed dust comae at optical and infrared wavelengths, but acknowledge that other molecules may be participating in less significant ways.

Given the large role CO plays in 29P/SW1's gas coma, it is worth revisiting what observational clues we have to its behavior. At high spectral resolution, the $\mathrm{CO}$ line profile has a complicated structure with a strong blueshifted velocity component, a weaker redshifted component, and a lowintensity skirted feature. A model containing a mix of sunward and nightside emission of $\mathrm{CO}$, along with an extended icy grain coma matches this line profile well (Crovisier et al. 1995; Gunnarsson et al. 2002, 2008). The source of sunward emission is thought to be fairly close to the nucleus surface near the subsolar point to provide the continuous blueshifted outgassing. This hypothesis is also supported by a recent updated model of dust outbursts for 29P (Schambeau 2018). Although the spectra are relatively low resolution, the overall line shape of the data is blueshifted by a small amount and is consistent with arising from a cold, slightly blueshifted and asymmetric gas, as reported from other observers (see Figure 1).

The narrow line profile of a cold gas may be a common feature in $\mathrm{CO}$ emission from many distantly active Centaurs and comets (Womack et al. 2017). In addition to Centaur 29P/ SW1 ( $~ 6 \mathrm{au})$, this characteristic was documented in Centaurs $174 \mathrm{P} /$ Echeclus at $\sim 6$ au (Wierzchos et al. 2017) and $95 \mathrm{P} /$ Chiron at 8.5 au (Womack \& Stern 1999), and comets C/1995 O1 (Hale-Bopp) beyond 4 au (Jewitt et al. 1996; Womack et al. 1997), C/2016 R2 (PanSTARRS) at 3 au (Biver et al. 2018; Wierzchos \& Womack 2018), and C/1997 J2 (MeunierDupouy) at $6.3 \mathrm{au}$ (Biver 2001). Given the similarity of $\mathrm{CO}$ emission profiles in the Centaurs and distantly active comets observed so far, future observations, especially with simultaneous measurements of the dust coma, will be invaluable for constraining models of the volatile and refractory structure of the nuclei, and ultimately to their formation environment.

Interestingly, the Centaur's quiescent dust coma measurably brightened over these three years: $m(1, r, 0)$ was 0.4 mag brighter during 2018-2019 (12.9 at $r=5.76$ au) than in 2016 (12.5 at $r=5.95 \mathrm{au}$ ). This difference can be seen in Figure 2 and is consistent with the baseline level of dust activity increasing $\sim 45 \%$ over the three years. During this time the CO quiescent level production rate increased by $\sim 24 \%$, which is within the measured uncertainties, and thus is also consistent with remaining steady. Given the uncertainties of the $\mathrm{CO}$ measurements, we cannot test the model that predicts that the dust and gas should change linearly during the quiescent stage (Enzian et al. 1997). The Centaur was only about 3\% closer to the Sun during 2018-2019, which is not large enough to attribute the increase solely to insolation change, which should vary as $r^{-2}$. Additional measurements are needed to confirm whether this change is part of a long-term brightening trend or just due to the variable nature of the dust production's quiescent stage (including possible contributions from nucleus rotation).

\subsection{Outbursts of $\mathrm{CO}$ and Dust}

Dust outbursts frequently brighten 29P/SW1's coma by several magnitudes within a few hours and generally resolve in under a week. Despite 29P/SW1's nearly circular path, the observing geometry (specifically the geocentric distance and phase angle) can contribute apparent changes of $\sim 1.5 \mathrm{mag}$, but it takes several months, not days, to achieve such an effect, and we have already taken steps to correct this in Section 3. The telescope pointing errors were less than $2^{\prime \prime}$ during the $\mathrm{CO}$ observations, which is not large enough to significantly affect the measured fluxes. Thus, the dust outbursts for which 29P/ SW1 is so well known and the rarely documented CO outbursts both require physical causes to explain their measured characteristics.

The nuclei of comets and Centaurs are assumed to be porous media containing a mixture of dust particles and volatile ices entrapped in adsorption sites (Klinger et al. 1996). Some models (e.g., Espinasse et al. 1991; Gunnarsson et al. 2008) assume the nucleus is composed substantially of amorphous water-ice containing trapped highly volatile gases, like $\mathrm{CO}$ or $\mathrm{CO}_{2}$, whose release is triggered by phase change of crystallization of the amorphous water-ice (similar to models of HaleBopp activity at large distances, e.g., Prialnik 1997). At 29P/ SW1's distance from the Sun, the effective blackbody surface temperature is $\sim 120 \mathrm{~K}$, which meets the energy threshold for the amorphous-crystalline change (see Equation (1) from Womack et al. 2017). Once released, gases may travel different paths out of the nucleus depending on their volatilities, some perhaps starting with sublimation from below the surface. In addition, other surface processes may trigger erosion and mass loss, which may lead to new release of gas without the simultaneous release of dust and vice versa (Enzian et al. 1997; Meech \& Svoren 2004; Prialnik et al. 2004; Steckloff \& Jacobson 2016). In addition, a recent updated model of dust outbursts in $29 \mathrm{P} / \mathrm{SW} 1$ is tied to the emission of CO from the sunward side (Schambeau 2018).

As Table 3 shows, there was no measurable change in either the Doppler shift or FWHM line width during the 2016 CO outburst. Thus, there is no observational evidence that the doubling of $\mathrm{CO}$ production arose from a change in the outgassing mechanism responsible for the quiescent stage (described in Section 4.1). We point out that Biver (1997) also reported a factor of $\sim 2$ increase in $Q(\mathrm{CO})$ in $29 \mathrm{P} / \mathrm{SW} 1$ that decayed in 2-3 days after the peak. Those data were obtained during 1995 November 15-19 with the Institut de Radioastronomie Millimétrique (IRAM) $30 \mathrm{~m}$ telescope. Thus, there are two documented cases of the development of a CO outburst in $29 \mathrm{P} / \mathrm{SW} 1$, and both lasted $\sim 4-5$ days.

The 2-3 day return to the quiescent level after the $\mathrm{CO}$ outburst is consistent with most of the molecules traveling out of the radio telescope beam after a few days. For example, consider that the SMT FWHM beam width at $230 \mathrm{GHz}$ is $32^{\prime \prime}$, which corresponds to a projected radius of $63,500 \mathrm{~km}$ at the comet's distance on 2018 November $24(\Delta=5.4 \mathrm{au})$. If we assume a CO expansion velocity of $0.3 \mathrm{~km} \mathrm{~s}^{-1}$ (consistent with all modeling of $\mathrm{CO}$ millimeter-wavelength spectra), then we estimate that molecules traveling parallel to the plane of the sky (zero blueshift to the geocentric velocity) would leave the beam in about 2.4 days $\left(t=x / v=6.35 \times 10^{4} \mathrm{~km} / 0.3 \mathrm{~km} \mathrm{~s}^{-1}=2.4\right.$ days), and more quickly with the smaller IRAM beam. Molecules traveling out of the plane of the sky would remain in the beam a little longer since their tangential velocity is reduced. Thus, the total $\sim 4-5$ day duration of the CO outbursts seen with the ARO SMT in 2016 (this paper) and with IRAM $30 \mathrm{~m}$ in 1995 (Biver 1997) are consistent with a very short-term (few hours) release of $\mathrm{CO}$ molecules from the nucleus and then most of the molecules moving out of the telescope beam. CO's 
lifetime against photodestruction is very long at this heliocentric distance and does not significantly reduce the $\mathrm{CO}$ coma size in a few days. Additional observations of $\mathrm{CO}$ outbursts in 29P/SW1 with higher temporal, spectral, and spatial resolution would significantly constrain model describing processes that affect $\mathrm{CO}$ emission at large distances from the Sun.

$\mathrm{CO}$ outgassing is often assumed to play an important role in the dust outbursts, largely due to its high production rate and the adequacy of some models to explain the quiescent activity (Cowan \& A'Hearn 1982; Enzian et al. 1997). In order to test the hypothesis that $\mathrm{CO}$ production is connected to the dust outbursts, we constructed the $Q(\mathrm{CO})$ and visible magnitude lightcurves. Intriguingly, the 2016 CO gas outburst was not immediately accompanied by an increase in dust production. The dust coma maintained a nearly constant quiescent magnitude of $m(1, r, 0)=12.9 \pm 0.2$ for at least 10 days after the $\mathrm{CO}$ outburst (Figure 2). Thus, either the $\mathrm{CO}$ outburst led to little to no increase in dust production, or it created a dust outburst after a $\sim 10$ day delay. Furthermore, our analysis also indicates that $\mathrm{CO}$ outbursts are not required to generate dust outbursts (see outburst B in Figure 2). The previously mentioned 1995 CO outburst reported by Biver (1997) did not have simultaneous measurements of the dust coma; thus, these 2016 data are the only known set of CO outburst in 29P/ SW1 with quasi-simultaneous information about the dust.

The two CO gas outburst patterns are similar to some of the visible outburst patterns in that they have a rapid rise with a slow decline. Both $\mathrm{CO}$ gas outbursts appeared to decay to quiescent levels a day or two more quickly (at least) than typical dust outbursts. When documented with lightcurves, dust outbursts appear to return to quiescent values in 3-5 days, sometimes longer (Trigo-Rodríguez et al. 2008, 2010; Miles et al. 2016). The longer decay time for dust can be explained by the dust having a lower expansion velocity than the gas (Schambeau 2018).

There are four noticeable dust outbursts during the observing campaign. Two occurred without a substantial increase in CO production (labeled $\mathrm{B}$ and $\mathrm{C}$ in Figure 2), and two may have coincided with a rise in $\mathrm{CO}$ (A and D). Here we describe and discuss all four in detail. On 2016 March 14, 29P/SW1 underwent a $\sim 3$ mag dust outburst (labeled $\mathrm{A}$ in Figure 2), which corresponds to an approximately sixteen-fold increase in brightness. After peak brightness, the coma steadily dimmed over weeks to its quiescent value, a decay time much longer than the other dust outbursts in this paper and reported elsewhere (e.g., Trigo-Rodríguez et al. 2008; Miles et al. 2016). We also consider that the lightcurve pattern for A may be due to two or more outbursts occurring within a few days of each other.

The second outburst, B, brightened by $\sim 3.3$ mag (a twentyfold increase) on 2018 November 22, which it maintained for a few days before decaying. As Figure 2 shows, $52 \mathrm{hr}$ after the start of this outburst, when the dust outburst was still at its highest point, the $\mathrm{CO}$ production rate was at the quiescent value. Therefore, dust outburst $\mathrm{B}$ appears to have been triggered, and even maintained over $\sim 2$ days, without an increase in the $\mathrm{CO}$ production rate.

Outburst $\mathrm{C}$ began on 2018 December 9, increased brightness by $\Delta m=2.5 \mathrm{mag}$, and 2.5 days later was accompanied by a slightly elevated $\mathrm{CO}$ production rate. Hypothetically, if the dust outburst mechanism for $\mathrm{B}$ and $\mathrm{C}$ were tied to a significant release of $\mathrm{CO}$, then such a $\mathrm{CO}$ outburst must have resolved itself within 1-2 days, i.e., more quickly than the observed $\mathrm{CO}$ outburst. This is a further indication of either no $\mathrm{CO}$ involvement with a dust outburst, or perhaps a scenario where two other short-lived $\mathrm{CO}$ outbursts occurred when we were not collecting data with the SMT. Regardless, whatever the mechanism was behind the $\mathrm{B}$ and $\mathrm{C}$ outbursts, they did not lead to a multiple-day-long increase of the $\mathrm{CO}$ emission.

The smallest dust outburst, labeled D, which peaked on 2019 January 8 with $\Delta m=1.1$ magnitude, does not have the same profile as the first three outbursts. Instead of a rapid brightening over a matter of hours, it appears to more gradually brighten over several days and then decay somewhat symmetrically, possibly indicating a different physical process at work. Also, outburst $\mathrm{D}$ coincides with a similar increase in $Q(\mathrm{CO})$, and there is some indication that both the gas and dust returned to quiescent levels a week later. Unfortunately, there are not enough measurements of the $\mathrm{CO}$ emission to further explore the gas relationship to the dust for this outburst. This is a further demonstration of the need to obtain more time-series observations of $\mathrm{CO}$ spectra along with measurements of the dust coma.

The data presented in this paper show that only two of the dust outbursts, A and D, may have been associated with an increased $\mathrm{CO}$ production rate. The paucity of $\mathrm{CO}$ measurements around the start of the dust outburst A makes drawing conclusions difficult, but there is some evidence for $\mathrm{CO}$ involvement, as the $Q(\mathrm{CO})$ and visible magnitude decline at similar rates during the weeks after the outburst, particularly on March 21-31. This similar apparent rate of decline could be also consistent with an extended source of $\mathrm{CO}$ from the coma grains at this time (e.g., Gunnarsson et al. 2003). There was no such evidence for an extended source in the CO outburst that occurred a few weeks earlier. In contrast, the CO outburst of early 2016 did not lead to an increased dust production for at least 10 days, and neither dust outburst B or C were associated with an increased $\mathrm{CO}$ production rate. Thus, $\mathrm{CO}$ and dust outbursts are not always correlated, and we may be observing $\mathrm{CO}$ arising from both the nucleus and an extended source in the coma.

The lack of correlation between the $\mathrm{CO}$ and dust outbursts in the data may be explained if $\mathrm{CO}$ is not substantially incorporated in a material with the dust component in the nucleus, or if the surface is regularly interrupted. Other possibilities to explain the separate dramatic release of large quantities of gas and dust is if the $\mathrm{CO}$ is primarily released through a highly porous material, or if another relatively minor volatile plays a large role in the dust outbursts.

We briefly consider other volatile candidates that might be involved in triggering the dust outbursts. A common candidate proposed for activity in many distantly active comets is $\mathrm{CO}_{2}$ (Ootsubo et al. 2012; Bauer et al. 2015; Womack et al. 2017). In particular, Gunnarsson et al. (2008) suggest that $\mathrm{CO}_{2}$ (with its higher atomic weight) might be mass-loading with $\mathrm{CO}$ in 29P/SW1 and slowing down its exit into the coma. However, using simultaneous spectroscopy from the Akari infrared telescope, $\mathrm{CO}_{2}$ has a very low measured upper limit of $Q\left(\mathrm{CO}_{2}\right)<3.5 \times 10^{26} \mathrm{~mol} \mathrm{~s}^{-1}$ in $29 \mathrm{P} / \mathrm{SW} 1$, corresponding to a mass-loss rate of $d M / d t<25 \mathrm{~kg} \mathrm{~s}^{-1}$ and a mixing ratio of $Q\left(\mathrm{CO}_{2}\right) / Q(\mathrm{CO})<0.01$ (Ootsubo et al. 2012). Measurements of the aggregate emission from $\mathrm{CO}_{2}+\mathrm{CO}$ at $4.5 \mu \mathrm{m}$ were made with photometry that are also consistent with very little $\mathrm{CO}_{2}$ in this Centaur (O. Harrington Pinto et al. 2020, in preparation) 
This is well below the predicted value $\mathrm{CO}_{2}$ in Gunnarsson et al. (2008) and thus the data are not consistent with significant mass-loading of $\mathrm{CO}_{2}$ with $\mathrm{CO}$, or with the dust outbursts, unless only a very minimal amount $\left(<25 \mathrm{~kg} \mathrm{~s}^{-1}\right)$ is needed.

As for other possible candidates for outbursting activity, $\mathrm{C}_{2} \mathrm{H}_{2}$ and $\mathrm{NH}_{3}$ also have relatively low sublimation temperatures (5 and $78 \mathrm{~K}$ respectively; Yamamoto 1985) and are detected in other comets with low abundances. They have not been identified in 29P/SW1, but their upper limits are not very constraining, $Q\left(\mathrm{C}_{2} \mathrm{H}_{2}\right)<117 \mathrm{~kg} \mathrm{~s}^{-1}$ and $Q\left(\mathrm{NH}_{3}\right)<309 \mathrm{~kg} \mathrm{~s}^{-1}$ (Table 2), so efforts to observe these two molecules may be fruitful. Other cosmogonically abundant species with low sublimation temperatures already have very low upper limits placed on them, and are unlikely to significantly compete with $\mathrm{CO}$ in the coma (see Table 2).

The dust outbursts in the lightcurve demonstrate an important point about the sporadic nature of the outbursts. $29 \mathrm{P} / \mathrm{SW} 1$ 's rotation period is in great disagreement between different authors with values ranging between $14 \mathrm{hr}$ and 50 days (Whipple 1980; Jewitt 1990; Meech et al. 1993; TrigoRodríguez et al. 2010; Ivanova et al. 2012; Schambeau 2018). This is a substantial problem, because an accurate estimate of $29 \mathrm{P} / \mathrm{SW} 1$ 's rotation period is needed to model its activity in more detail. Trigo-Rodríguez et al. (2010) partly favor a rotation period of $\sim 50$ days because of the stated annual average of $\sim 7$ dust outbursts/year, which corresponds to an average of one outburst every $\sim 50$ days. However, this average time between outbursts carries a high dispersion: many $>1$ mag outbursts occur only 1-2 weeks apart (and not $\sim 50$ days) as the data in Figure 2 show. Even the quiescent stage is highly variable over days to weeks, which makes it difficult to establish the start and end times of dust outbursts. Furthermore, some dust outbursts may overlap in time, which may be the case for the outburst A. Thus, as Trigo-Rodríguez et al. (2010) point out, the $\sim 50$ day average obtained from an observed seven outbursts/year may not be physically tied to the actual rotation rate of the nucleus.

In order to constrain the mechanisms driving the activity of this enigmatic Centaur, further observations of 29P/SW1 at all wavelengths with myriad techniques are strongly encouragedespecially simultaneously. 29P/SW1 recently underwent perihelion in 2019 March, and it has now entered the epoch when its activity historically increases (Cabot et al. 1996; Krisandova \& Svoren 2014). 29P/SW1's activity level increased significantly after its orbit changed due to a close interaction with Jupiter in 1975. In 2038, 29P/SW1 will have another important close approach with Jupiter that is predicted to double its eccentricity (Sarid et al. 2019). 29P/SW1's activity level may very well be affected by this new orbit change and this is another motivation for closely monitoring the Centaur in the next few decades. Continuous longterm ( $>80$ days) monitoring of the $\mathrm{CO}$ production rate, preferably with high spectral resolution, is needed to document any increases in $Q(\mathrm{CO})$ that lead to dust outbursts, and if so, the evolution and relationship of the two phenomena. Time-series monitoring of other molecular species will also be useful to constraining improved models of the dust outbursts. A world-wide observing campaign (http://wirtanen.astro.umd.edu/29P/29P_obs.shtml) for 29P/SW1 was initiated in 2018 by M. Womack, G. Sarid, and T. Farnham. Participation is strongly encouraged as it may help different teams coordinate observing runs and increase the chances of obtaining simultaneous measurements and maximizing scientific return.

\section{Summary and Conclusions}

For years it has been hypothesized that $\mathrm{CO}$ outgassing stimulates the release of dust outbursts in 29P/SW1, as well as the quiescent dust coma. Comparison of the $Q(\mathrm{CO})$ and visible lightcurve data, an effective tracer of dust production in the Centaur, show that the relationship between outbursts of dust and gas is not always this straightforward.

Throughout 21 days of CO observations over three years, 29P/ SW1's quiescent gas production rate changed from $Q(\mathrm{CO})=$ $(2.9 \pm 0.2) \times 10^{28} \mathrm{~mol} \mathrm{~s}^{-1}$ to $(3.6 \pm 0.7) \times 10^{28} \mathrm{~mol} \mathrm{~s}^{-1}$. This is an increase of $\sim 24 \%$, but is also consistent with remaining steady within the uncertainties. The quiescent value of the heliocentric magnitude corrected for phase angle brightened by $\sim 45 \%$ from $m$ $(1, r, 0)=12.9 \pm 0.2$ to $12.5 \pm 0.2$, while the heliocentric distance decreased by only $4 \%$.

Several outbursts were recorded during the observing periods. On 2019 February 25.7 the $\mathrm{CO}$ production rate doubled within $70 \mathrm{hr}$ and then returned to the original quiescent value approximately three days later. The $\mathrm{CO}$ emission line profile characteristics appeared typical of what is observed for $\mathrm{CO}$ in distant comae and showed no evidence for substantial deviation related to the outbursting mechanism. The CO increase was unmatched by a change in dust production for at least 10 days, consistent with no significant dust involvement as a consequence of the $\mathrm{CO}$ outburst.

Four dust outbursts were recorded at visible wavelengths: two (B on 2018 November 22 and C on 2018 December 9) occurred without a measurable increase in $\mathrm{CO}$ production and two (A on 2016 March 14 and D on 2019 January 8) coincided with increased $\mathrm{CO}$ amounts. The lack of a strong correlation of gas and dust behavior during all of the $\mathrm{CO}$ and dust outbursts shows that although the $\mathrm{CO}$ and dust production increases may be sometimes coincident, they are not always significantly entrained in $29 \mathrm{P} / \mathrm{SW} 1$. The data are not consistent with the commonly held assumption about 29P/SW1 that increased CO outgassing must always be involved with the dust outbursts and may provide important observational constraints for cometary outbursting models.

The dust outburst lightcurve shapes may hold additional clues to the mechanisms involved: outbursts A, B, and C had an asymmetric shape with a steep build-up and gradual decay, whereas outburst D appeared more symmetric about the peak including a more gradual build-up and decay. Outburst D (which also coincided with elevated $\mathrm{CO}$ production) appears to have been triggered with a less explosive process than the other three, and it is also the smallest outburst observed of this group. Additional coordinated observations of multiple species in the comae of $29 \mathrm{P} / \mathrm{SW} 1$ are needed to further test models of the interplay of the gas and dust components in driving the quiescent activity and outbursts.

This material is based on work supported by the National Science Foundation under grants No. AST-1615917 and AST1945950 (M.W. as PI). K.W. was partially supported from the University of South Florida Duckwall Summer Research Fellowship. The authors are grateful to an anonymous referee who provided a critical reading of the manuscript and helpful comments. The authors also thank the ARO $10 \mathrm{~m}$ SMT staff, 
the Minor Planet Center, and the observers who contribute to the Lesia database of comet observations. The SMT is operated by the ARO, the Steward Observatory, and the University of Arizona, with support through the NSF University Radio Observatories program grant AST-1140030.

\section{ORCID iDs}

K. Wierzchos (D) https://orcid.org/0000-0002-4884-9367

M. Womack (D) https://orcid.org/0000-0003-4659-8653

\section{References}

Bauer, J. M., Stevenson, R., Kramer, E., et al. 2015, ApJ, 814, 85 Bieler, A., Altwegg, K., Balsiger, H., et al. 2015, Natur, 526, 678 Biver, N. 1997, PhD thesis, Univ. Paris

Biver, N. 2001, ICQ, 23, 85

Biver, N., Bockelée-Morvan, D., Crovisier, J., et al. 1999, AJ, 118, 1850

Biver, N., Bockelée-Morvan, D., Paubert, G., et al. 2018, A\&A, 619, A127

Bockelee-Morvan, D., Biver, N., Opitom, C., et al. 2014, in Asteroids, Comets, Meteors 2014, ed. K. Muinonen et al. (Helsinki: ACM), 49

Cabot, H., Enzian, A., Klinger, J., \& Majolet, S. 1996, P\&SS, 44, 1015

Cochran, A., Barker, E. S., \& Cochran, W. 1980, AJ, 85, 474

Cochran, A. L., \& Cochran, W. D. 1991, Icar, 90, 172

Cochran, A. L., Cochran, W. D., Barker, E. S., \& Storrs, A. D. 1991, Icar, 92, 179

Cowan, J. J., \& A'Hearn, M. F. 1982, Icar, 50, 53

Crovisier, J., Biver, N., Bockelee-Morvan, D., et al. 1995, Icar, 115, 213

Crovisier, J., \& Le Bourlot, J. 1983, A\&A, 123, 61

Dones, L., Brasser, R., Kaib, N., \& Rickman, H. 2015, SSRv, 197, 191

Enzian, A., Cabot, H., \& Klinger, J. 1997, A\&A, 319, 995

Espinasse, S., Klinger, J., Ritz, C., \& Schmitt, B. 1991, Icar, 92, 350

Feldman, P. D., McPhate, J. B., Weaver, H. A., Tozzi, G.-P., \& A'Hearn, M. F 1996, AAS DPS Meeting, 28, 1084

Fernández, J. A., Tancredi, G., Rickman, H., \& Licand ro, J. 1999, A\&A, 352,327

Ferrín, I. 2010, P\&SS, 58, 365

Festou, M. C., Gunnarsson, M., Rickman, H., Winnberg, A., \& Tancredi, G. 2001, Icar, 150, 140

Fulle, M. 1992, Natur, 359, 42

Fulle, M., Blum, J., \& Rotundi, A. 2019, ApJL, 879, L8

Fulle, M., Cremonese, G., \& Böhm, C. 1998, AJ, 116, 1470

Gronkowski, P. 2004, AN, 325, 343

Gunnarsson, M., Bockelée-Morvan, D., Biver, N., et al. 2008, A\&A, 484, 537

Gunnarsson, M., Bockelée-Morvan, D., Winnberg, A., et al. 2003, A\&A, 402, 383

Gunnarsson, M., Rickman, H., Festou, M. C., Winnberg, A., \& Tancredi, G. 2002, Icar, 157, 309

Haser, L. 1957, BSRSL, 43, 740

Hosek, M. W., Jr., Blaauw, R. C., Cooke, W. J., \& Suggs, R. M. 2013, AJ, 145,122

Hughes, D. W. 1975, QJRAS, 16, 410

Ivanova, A. V., Afanasiev, V. L., Korsun, P. P., et al. 2012, SoSyR, 46, 313

Ivanova, O., Agapitov, O., Odstrcil, D., et al. 2019, MNRAS, 486, 5614

Ivanova, O. V., Luk'yanyk, I. V., Kiselev, N. N., et al. 2016, P\&SS, 121, 10
Ivanova, O. V., Skorov, Y. V., Korsun, P. P., Afanasiev, V. L., \& Blum, J. 2011, Icar, 211, 559

Jewitt, D. 1990, ApJ, 351, 277

Jewitt, D., Senay, M., \& Matthews, H. 1996, Sci, 271, 1110

Jockers, K., Bonev, T., Ivanova, V., \& Rauer, H. 1992, A\&A, 260, 455

Klinger, J., Levasseur-Regourd, A.-C., Bouziani, N., \& Enzian, A. 1996, P\&SS, 44, 637

Korsun, P. P., Ivanova, O. V., \& Afanasiev, V. L. 2008, Icar, 198, 465

Kossacki, K. J., \& Szutowicz, S. 2013, Icar, 225, 111

Krisandova, Z., \& Svoren, J. 2014, in Asteroids, Comets, Meteors 2014, ed K. Muinonen et al. (Helsinki: ACM), 291

Larson, S. M. 1980, ApJL, 238, L47

Larson, S. M., Edberg, S. J., \& Levy, D. H. 1991, ASSL, 167, 209

Levison, H. F., \& Duncan, M. J. 1994, Icar, 108, 18

Levison, H. F., \& Duncan, M. J. 1997, Icar, 127, 13

Luspay-Kuti, A., Mousis, O., Lunine, J. I., et al. 2018, SSRv, 214, 115

Mandt, K. E., Mousis, O., Marty, B., et al. 2015, SSRv, 197, 297

Meech, K. J., Belton, M. J. S., Mueller, B. E. A., Dicksion, M. W., \& Li, H. R. 1993, AJ, 106, 1222

Meech, K. J., \& Svoren, J. 2004, in Comets II, ed. M. C. Festou, H. U. Keller, \& H. A. Weaver (Tucson, AZ: Univ. Arizona Press), 317 Miles, R. 2016, Icar, 272, 387

Miles, R., Faillace, G. A., Mottola, S., et al. 2016, Icar, 272, 327

Mumma, M. J., \& Charnley, S. B. 2011, ARA\&A, 49, 471

Ootsubo, T., Kawakita, H., Hamada, S., et al. 2012, ApJ, 752, 15

Paganini, L., Mumma, M. J., Boehnhardt, H., et al. 2013, ApJ, 766, 100

Prialnik, D. 1997, ApJL, 478, L107

Prialnik, D., \& Bar-Nun, A. 1990, ApJ, 363, 274

Prialnik, D., Benkhoff, J., \& Podolak, M. 2004, in Comets II, ed. M. C. Festou, H. U. Keller, \& H. A. Weaver (Tucson, AZ: Univ. Arizona Press), 359

Richter, N. 1941, AN, 271, 207

Roemer, E. 1958, PASP, 70, 272

Roemer, E. 1962, PASP, 74, 351

Rubin, M., Altwegg, K., van Dishoeck, E. F., \& Schwehm, G. 2015, ApJ, 815, L11

Sarid, G., Volk, K., Steckloff, J. K., et al. 2019, ApJ, 883, L25

Schambeau, C. 2018, PhD thesis, Univ. Central Florida, Orlando

Schambeau, C. A., Fernández, Y. R., Samarasinha, N. H., Mueller, B. E. A., \& Woodney, L. M. 2017, Icar, 284, 359

Schambeau, C. A., Fernández, Y. R., Samarasinha, N. H., Woodney, L. M., \& Kundu, A. 2019, AJ, 158, 259

Schleicher, D. G., \& Bair, A. N. 2011, AJ, 141, 177

Senay, M. C., \& Jewitt, D. 1994, Natur, 371, 229

Shi, J. C., Ma, Y. H., \& Zheng, J. Q. 2014, MNRAS, 441, 739

Steckloff, J. K., \& Jacobson, S. A. 2016, Icar, 264, 160

Taquet, V., van Dishoeck, E. F., Swayne, M., et al. 2018, A\&A, 618, A11

Trigo-Rodríguez, J. M., García-Hernández, D. A., Sánchez, A., et al. 2010 , MNRAS, 409, 1682

Trigo-Rodríguez, J. M., García-Melendo, E., Davidsson, B. J. R., et al. 2008, A\&A, 485, 599

Wesołowski, M., \& Gronkowski, P. 2018, EM\&P, 121, 105

Whipple, F. L. 1980, AJ, 85, 305

Wierzchos, K., \& Womack, M. 2018, AJ, 156, 34

Wierzchos, K., Womack, M., \& Sarid, G. 2017, AJ, 153, 230

Womack, M., Festou, M. C., \& Stern, S. A. 1997, AJ, 114, 2789

Womack, M., Sarid, G., \& Wierzchos, K. 2017, PASP, 129, 031001

Womack, M., \& Stern, S. A. 1999, SoSyR, 33, 187

Yamamoto, T. 1985, A\&A, 142, 31 